\title{
Análisis confirmatorio de la Escala de Autoevaluación Breve de Bienestar y Autonomía en Adultos Mayores de Coahuila y Yucatán, México*
}

\author{
Confirmatory Analysis of the Short Self-Assessment of \\ Wellbeing and Autonomy Scale in Older Adults in Coahuila and \\ Yucatán, Mexico
}

José González Tovar** ORCID: 0000-0002-2507-5506

Ana Laura Carrillo Cervantes ORCID: 0000-0003-2920-4675

Universidad Autónoma de Coahuila, México

Antonio Yam Sosa ORCID: 0000-0002-7499-1009 Universidad Autónoma de Yucatán, México

Daniel Sifuentes Leura

ORCID: 0000-0002-4851-1593

Universidad Autónoma de Coahuila, México

Patricia Isolina del Socorro Gómez Aguilar ORCID: 0000-0001-7196-1544 Universidad Autónoma de Yucatán, México

Recibido: 24 de septiembre de 2019 Revisado: 7 de diciembre de 2019 Aceptado: 19 de febrero de 2020

\section{Resumen}

El objetivo de este trabajo fue realizar un Análisis Factorial Confirmatorio de la Escala de Bienestar y Autonomía específica para adultos mayores en dos muestras mexicanas, lo que permitió determinar la validez del constructo medido. Se utilizó un diseño cuantitativo, transversal con alcances explicativos. Se analizó una muestra incidental de 895 adultos mayores, de los cuales $59.8 \%$ eran habitantes de la ciudad de Saltillo, Coahuila, México y $40.2 \%$ de Tizimín, Yucatán, México. Se utilizó la Escala de Autoevaluación Breve de Bienestar y Autonomía para Adultos Mayores. Se procesó el Análisis Factorial Exploratorio, Paralelo y Confirmatorio con el método de Mínimos Cuadrados Generalizados, además de obtener la confiabilidad y los estadísticos descriptivos de las dimensiones. La estructura que se identificó presentó indicadores de ajuste estadístico adecuado. Originalmente, la estructura consideraba dos dimensiones: la autonomía y el bienestar, sin embargo, los resultados mostraron la separación en tres dimensiones de la medición de bienestar.

Palabras clave: adulto mayor, autonomía, bienestar, análisis confirmatorio.

Artículo de investigación. Citar como: González, T. J., Carrillo, C. A. L., Yam, S. A., Sifuentes, L. D. y Gómez, A. P. I. (2020). Análisis confirmatorio de la Escala de Autoevaluación Breve de Bienestar y Autonomía en Adultos Mayores de Coahuila y Yucatán, México. Diversitas: Perspectivas en Psicología, 16(2), 297-309. https://doi.org/10.15332/22563067.6296

** Autor de correspondencia: José González Tovar. Correo electrónico: josegonzaleztovar@uadec.edu.mx. Dirección postal: Departamento de Posgrado, Facultad de Psicología Unidad Saltillo, Unidad Campo Redondo, Edificio D Planta Baja, Universidad Autónoma de Coahuila, México 


\section{Abstract}

The objective of this work was to carry out a Confirmatory Factorial Analysis of the Scale of Wellbeing and Specific Autonomy for Older Adults in two Mexican samples, which allowed to establish the validity of the measured construct. A quantitative, transversal design with explanatory scopes was used. An incidental sample of 895 aging adults was obtained, from which $59.8 \%$ were inhabitants of the Saltillo city, Coahuila, Mexico, and 40.2\%, of Tizimín city, Yucatán, Mexico. We used the Short SelfAssessment of Wellbeing and Autonomy for Older Adults. The Exploratory, Parallel and Confirmatory Factor Analysis was processed with the Generalized Least Squares Method, in addition to obtaining the reliability and descriptive statistics of dimensions. The identified structure showed adequate statistical adjustment indicators. Initially, the structure considered two dimensions: autonomy and wellbeing; however, the results showed the separation in three dimensions of the wellbeing measurement.

Keywords: elderly, autonomy, well-being, confirmatory analysis.

\section{Introducción}

Los estudios sobre el cambio y expectativa de vida de la población han determinado que, tanto de forma absoluta como relativa, se ha incrementado el número de adultos mayores en las últimas décadas con respecto a la población menor de 60 años (Pelcastre-Villafuerte, Treviño-Siller, GonzálezVázquez y Márquez-Serrano, 2011). Este fenómeno está relacionado directamente con el aumento en la expectativa de vida e indirectamente con el descenso en la natalidad (Alvarado-García, LampreaReyes y Murcia-Tabares, 2017; Loredo-Figueroa, Gallegos-Torres, Xeque-Morales, Palomé-Vega y Juárez-Lira, 2016). En este sentido, aunque es de esperar que las circunstancias de vida permitan a la población acrecentar sus años de vida, se prevé que esta situación se dé bajo condiciones cualitativas aún inciertas (Manrique-Espinoza et al., 2013), por lo que medición de dichas condiciones favorece una mayor certidumbre y permite evaluar políticas de atención a esta población.

Además, en términos sociales, con el aumento de la población de adultos mayores se espera una convivencia intergeneracional inédita. Los jóvenes convivirán con personas de muy avanzada edad, los adultos jóvenes se relacionarán con personas adultas mayores en circunstancias que obligará, en particular en la familia, a reflexionar y reconstruir las maneras de convivir y dar apoyo social. Además, la longevidad irá acompañada de la necesidad de contar con servicios de salud física, mental y de asistencia social en la etapa final de la vida con requerimientos sin precedentes, lo que hará necesaria la disposición en la ayuda y serán necesarias acciones que comprometerán al estado y la sociedad para enfrentar las crecientes y diversificadas necesidades (Lolas Stepke, 2001; Vivaldi y Barra, 2012).

En México, actualmente hay un cambio en la dinámica y composición de la población que ha dado como resultado un incremento de la esperanza de vida. En los años setenta, la media se ubicaba en menos de 70 años para varones y de 70 para las mujeres, para el año 2012 es de casi de 76 años para ambos sexos (Monroy-Rojas et al., 2016). En el 2014, la población de más 60 años era de 12.7 millones; además, de esa cantidad el $44.3 \%$ no recibía ingreso alguno por pensiones o seguridad social (Damián, 2016).

En el mismo sentido, la Organización Mundial de la Salud (OMS) calculó una cantidad de 629 millones de personas adultas mayores en el 2012. El pronóstico indica un aumento a casi 2,000 millones en el año 2050, con repercusiones en lo social, lo económico y lo político, además de la sobrecarga del sistema de cuidados de la salud, que deberá otorgar servicios 
y medicamentos a la población mayor con más necesidades y demandas (Bernal Reyes, Arias Díaz, Hormigó Puertas y Roselló Leyva, 2015; Cervantes Becerra, Villarreal Ríos, Galicia Rodríguez, Vargas Daza y Martínez González, 2015).

En conjunto con el aumento de la población de adultos mayores se necesita un aumento del cuidado específico, dado que la vulnerabilidad física de las personas adultas mayores complica su ejercicio en las acciones básicas de la vida diaria. Además, se encuentra un detrimento en su salud, su entorno social y su estado emocional, aspectos modelados por la carga cultural del contexto (González Tovar y Garza Sánchez, 2016; Melguizo Herrera, Acosta López y Castellano Pérez, 2012). Cada adulto mayor vive transformaciones en el ámbito físico, el apoyo social y la salud mental, lo que trastorna su bienestar y percepción de este (Loredo-Figueroa et al., 2016).

Dichas transformaciones, junto con las enfermedades crónicas y degenerativas, restringen la práctica de sus actividades, ya que estas circunstancias de morbilidad con consecuencias no deletéreos, son progresivas y arrastran a la restricción de sus ocupaciones, además limitan su colaboración social, notable para el adulto mayor ya que ha afianzado su identidad personal, en la cual las funciones de afecto y cuidado son transcendentales (JiménezAguilera, Baillet-Esquivel, Ávalos-Pérez y CamposAragón, 2016).

En este sentido, una de las condiciones comúnmente poco valoradas en las personas adultas mayores es el factor afectivo, mismo que es considerado como una característica de gran determinación de las condiciones de salud y calidad de vida. Se ha mostrado que la depresión tiene una correlación con una mayor morbilidad (Afilalo et al., 2012; Chen y Chen, 2017) y es considerada también un factor de riesgo de mayor mortalidad, ya que impacta de manera negativa aspectos funcionales, nutricionales y sociales, y dificulta los procesos de recuperación (Domínguez-Ardila y García-Manrique, 2014). Adicional a esto, se ha reportado una relación con enfermedades cardiacas y degenerativas que reducen la capacidad de actividad del adulto mayor (Karlsson, Magnusson, von Schewelov y Rosengren, 2013; Zheng et al., 2015). El estado de fragilidad producto del aumento de enfermedades se empeora por condiciones sociales y económicas desfavorables, condiciones de depresión, estrés, dependencia y perdida de funcionalidad (García, 2010).

Con respecto a la salud física, la calidad de vida y la salud en adultos mayores se ha estudiado utilizando mediciones de morbilidad y mortalidad, además de la esperanza de vida. Sin embargo, se desconocen otros aspectos como la percepción sobre su salud, el conocimiento de las redes de apoyo social, la protección social y los servicios de atención a la salud (Botero de Mejía y Pico Merchán, 2007).

Por otro lado, existe una amplia discusión acerca de la estimación del envejecimiento exitoso o saludable y el bienestar en los adultos mayores. En la conceptualización de bienestar personal se integran dimensiones como la satisfacción vital, la satisfacción presente que experimentan en su vida cotidianidad, la presencia de emociones positivas y la sensación de felicidad (Carmona Valdés y Ribeiro Ferreira, 2010).

La percepción de bienestar y calidad de vida puede verse afectada por la salud física, psicológica, su nivel de independencia y el apoyo social. Además, se ha considerado que vivir con calidad es poseer una existencia agradable o vivir una vida con condiciones satisfactorias (Pérez Vargas, 2015).

Además, la definición de calidad de vida abarca las dimensiones física, psicológica, social y percepción de la salud, la sensibilidad al dolor físico y sobre todo satisfacción con la vida. Sin embargo, los instrumentos para medir la calidad de vida no reemplazan al resto de las evaluaciones que contienen indicadores de tipo biológico, morfológico y sintomático, sino que las completan, y contribuyen al conocimiento que tiene el adulto mayor sobre su estado de salud (Bernal Reyes et al., 2015; CabezasLeón, García-Caballero y Morente-Matas, 2008).

Debido a lo anterior, se ha dado un incremento en el interés internacional sobre la medición y evaluación de la calidad de vida en el adulto mayor relacionado con conceptos de bienestar y envejecimiento exitoso. La literatura sobre la calidad de vida ha aumentado generando varios instrumentos para medir calidad de vida en adultos mayores (Haywood, 
Garratt y Fitzpatrick, 2005; Zaninotto, Falaschetti y Sacker, 2009). Estos instrumentos han permitido identificar factores esenciales para la calidad de vida, se destaca así la religión y el afrontamiento orientado a la espiritualidad (Acevedo Alemán y González Tovar, 2014; Vitorino et al., 2016).

Por otro lado, el bienestar en el adulto mayor, en sus aspectos sociales, ha sido también relacionado con cambios en el estado nutricional, asociados con la medición de la soledad y redes de apoyo social (Maseda et al., 2018). Las relaciones sociales positivas pueden considerarse como un determinante en la salud de las personas mayores, la proximidad emocional, junto con la frecuencia y tiempo dedicado a la convivencia, también están correlacionados con la salud (Guerrero-López, Mendieta Cabrera, Lara-Muñoz y Ortiz García, 2017).

En relación con la autonomía, la calidad de vida está vinculada con actividades que se dan de forma natural, les permiten salvaguardar la salud física y es un factor protector significativo para la prevención de diversos padecimientos (Garza Sánchez y González Tovar, 2017; Ramírez-Villada, Chaparro-Obando, León-Ariza, y Salazar Pachón, 2015; Salazar Pachón, Ramírez Villada, Chaparro y León, 2014). Además, es relevante evaluar el impacto que la condición física tiene en la percepción de bienestar, estudios han encontrado que el dolor físico impacta de forma negativa en la autonomía e independencia funcional y la valoración de la calidad de vida de las personas adultas mayores, dando resultados negativos o adversos (Borda, Acevedo González, David, Morros González y Cano, 2016). Igualmente, a pesar de que la autonomía no puede medirse directamente existen modelos que permiten estimar un cálculo de esta capacidad (Sève-Ferrieu, 2017).

Se ha encontrado tambien que, un alto porcentaje de personas adultas mayores realizan con libertad sus actividades cotidianas relacionadas con el cuidado de la salud, movimientos y esfuerzos moderados, además, ocupan un rol físico que permite desarrollar sus actividades habituales sin restricciones o problemas (Marín Monroy y Castro Molinares, 2011), aunque otros estudios indican que la funcionalidad es dependiente de la presencia de enfermedades principalmente crónicas, sintomatología depresiva, apoyo social y nivel de escolaridad (Chang et al., 2010). Entre más comprometida se encuentre la salud tanto física como mental, será más la presencia de disfunción social, ansiedad y depresión (Urzúa, Pavlov, Cortés y Pino, 2011).

De manera complementaria, en los adultos mayores, la calidad de vida relacionada con la salud (CVRS) contiene indicadores de tipo biológico, psicológico y social, especialmente la capacidad para realizar las actividades de la vida diaria, la dificultad para el autocuidado y las situaciones de dependencia que impide disfrutar completamente de la vida familiar y de las redes de apoyo de carácter social (Cardona, 2010).

De la misma manera, el nivel y características de la percepción de la calidad de vida está modulado por valoraciones que la persona realiza tanto de las situaciones objetivas de su vida (e.g., hechos), como del nivel de satisfacción que percibe sobre las mismas (Urzúa y Caqueo-Urízar, 2012). Así, la utilización de instrumentos de medición en salud centrados en el bienestar de los adultos mayores puede ser un objeto de estudio tanto para la práctica clínica como también para las evaluaciones de salud en el grupo poblacional (Urzúa y Navarrete, 2013).

Estos antecedentes nos permiten delimitar la problemática del estudio, enfocada al diseño de herramientas que permitan mediciones objetivas del nivel de bienestar percibido del adulto mayor, que permita obtener un panorama de las condiciones en las que se encuentra esta población, además de valorar el impacto de políticas de salud pública que abarquen tanto aspectos físicos como mentales. Dicho esto, el objetivo de este trabajo fue realizar el Análisis Factorial Confirmatorio de una Escala de Bienestar y Autonomía específica para adultos mayores en dos muestras mexicanas, lo que permitirá obtener la validez del constructo medido.

\section{Método}

\section{Diseño}

Se utilizó un diseño de corte cuantitativo, con formato de ejecución transversal y con alcances en el análisis estadístico de carácter 
descriptivo-correlacional (Hernández Sampíeri, Fernández Collado y Baptista Lucio, 2014).

\section{Participantes}

El total de la muestra fue de 895 adultos, de los cuales $59.8 \%(n=535)$ eran habitantes de la ciudad de Saltillo del estado de Coahuila y el $40.2 \%$ $(\mathrm{n}=360)$ del municipio de Tizimín del estado de Yucatán, México. El 48.9\% $(n=438)$ fueron mujeres y $51.1 \%(n=457)$ hombres. El método de selección de los participantes fue mediante un muestreo por voluntarios. En cuanto a la escolaridad de los participantes, el 33.9\% $(n=303)$ tenían educación primaria, el $14.5 \%(n=130)$ secundaria, $10.7 \%(n=96)$ preparatoria o bachillerato, $7.6 \%(n=68)$, el nivel de licenciatura lo tenían el $17 \%$ ( $n=152$ ), en nivel posgrado, se encontraba el 3.5\% $(n=40)$. El $11.8 \%$ reportó no haber completado ningún nivel educativo. El 58.2\% ( $n=521)$ estaba casado, el $5.4 \%(n=48)$ declararon que eran solteros, $15.4 \%(n=138)$ divorciados, el $14.3 \%(n=128)$ eran viudos, $4.4 \%(n=39)$ se encontraban en unión libre y el $2.1 \%(n=19)$ eran separado.

\section{Instrumento}

Se utilizó la Escala Breve de Bienestar y Autonomía en Adultos Mayores diseñada a partir de la Escala de Calidad de Vida para Adultos Mayores en Comunidad (González Tovar y Garza Sánchez, 2016). Se consideraron dos dimensiones: la primera fue la Autonomía, que estuvo compuesta originalmente por siete reactivos que medían el nivel de satisfacción percibida para efectuar actividades cotidianas. La segunda fue el Bienestar, compuesto por 14 reactivos que evaluaron la autopercepción de satisfacción con las condiciones de vida físicas, emocionales y sociales. El formato de respuesta fue una escala ordinal con cinco opciones de respuesta que van en el caso del Bienestar de $0=$ nunca lo pienso o siento, hasta 4 = siempre lo pienso o siento. Para la Autonomía las opciones eran $0=$ Nada satisfecho, hasta $4=$ Muy satisfecho. La dimensión de Autonomía obtuvo un coeficiente alfa de Cronbach de 0.815 con una correlación promedio entre elementos de 0.262 y el Bienestar obtuvo un coeficiente alfa de 0.950 y correlación de 0.735 .

\section{Procedimiento}

El procedimiento de recolección de los datos de la muestra de Coahuila se llevó a cabo mediante una entrevista directa no remunerada en el Centro de Atención al Adulto Mayor de la ciudad de Saltillo (Coahuila). El tiempo de respuesta fue de aproximadamente 40 minutos. El consentimiento informado de los participantes se obtuvo en apego a los principios éticos de la Declaración de Helsinki (Mazzanti Di Ruggiero, 2011). La muestra de Yucatán se obtuvo del Centro de Salud Urbano (csu) del municipio de Tizimín, se revisó el registro de las Adultos Mayores con el fin de identificar a todos aquellos que estaban inscritos en los 12 módulos. El procedimiento para la administración del instrumento fue en el domicilio de cada participante, se solicitó la firma de la hoja de consentimiento informado, la duración aproximada en la administración del instrumento fue de 25 minutos.

\section{Análisis de datos}

Los datos se exploraron mediante estadísticos descriptivos, medidas de tendencia central, de dispersión y medidas de distribución. Las variables con coeficientes de asimetría o curtosis superiores a dos puntos fueron descartadas para posteriores procedimientos (Bandalos y Finney, 2010; Lloret-Segura, Ferreres-Traver, Hernández-Baeza, y Tomás-Marco, 2014). Se procesó el Análisis Factorial Exploratorio (AFE) con 448 casos que correspondieron al $50 \%$ de la base de datos, se utilizó el método de Mínimos Cuadrados No Ponderados (ULS), con una rotación oblicua mediante el método Promax. Como criterios para la extracción de los factores se utilizaron dos, el criterio de Káiser de autovalores iguales o mayores a uno. El segundo criterio fue el Análisis Paralelo (AP), mismo que se procesó con el mismo método que el AFE. Se utilizó el AP con implementación óptima (Timmerman y Lorenzo-Seva, 2011). Con este análsis se obtuvieron los índices de Simplicidad de Bentler (1977), cuyos valores adecuados son los cercanos a uno (Fleming y Merino Soto, 2005) y de Simplicidad de la Extracción (Lorenzo-Seva, 2003) así como el índice $H$ de replicabilidad del constructo, cuyos valores deben de ser iguales o mayores a 0.80 para indicar una variable latente bien definida (Rodriguez, Reise, y Haviland, 2016). 
El Análisis Factorial Confirmatorio (AFC) se procesó con 447 casos, el método de Mínimos Cuadrados Generalizados, se obtuvieron los Índices de Bondad de Ajuste general (GFI) y corregido de Jöreskog (AGF), el Error Cuadrático Medio de Aproximación (RMSEA) y la Raíz del Residuo Medio Estandarizado (RMR).

En cuanto a la confiabilidad, esta se obtuvo por dos métodos, el coeficiente alfa de Cronbach y el coeficiente alfa Ordinal, dado que el nivel de medición de las variables también puede ser considerado como categórico ordinal (Oliden y Zumbo, 2008). Se utilizó el software estadístico SPSS 25, Factor 10 y Excel para las estimaciones.

\section{Resultados}

Previo al procesamiento se analizaron los supuestos para la ejecución del análisis factorial exploratorio. La prueba de esfericidad de Barttlet indicó buena adecuación de la matriz de correlaciones (кмо=.917), además, la prueba de esfericidad de Bartlett rechazó la hipótesis de independencia entre las variables $\left(\chi^{2}=11458.527 ; p<0.001\right)$.

En el AP, se obtuvo una recomendación para una estructura de dos factores, sin embargo, al procesar también una solución de cuatro factores, el Índice de Simplicidad de Bentler de fue S=.931 (IC95\%=.881968), el índice de Simplicidad de la Extracción fue de LS $=.503$ (IC95\%=.451-.584), valores que se considerad

Tabla 1.

Estadísticos descriptivos para la Autoevaluación Breve de Bienestar y Autonomía en Adultos Mayores

\begin{tabular}{|c|c|c|c|c|}
\hline Autopercepción de: & M & $\mathrm{DE}$ & As & k \\
\hline BG1. Su estado de salud en general & 2.73 & 0.880 & -0.573 & 0.469 \\
\hline BG2. Su estado de ánimo & 2.94 & 0.805 & -0.571 & 0.498 \\
\hline BG3. Su salud física & 2.64 & 0.790 & -0.123 & -0.202 \\
\hline BG4. Sus relaciones sociales & 2.83 & 0.907 & -0.777 & 0.810 \\
\hline BG5. Su relación con familiares & 3.10 & 0.900 & -0.907 & 0.575 \\
\hline BG6. Se enferma con mayor frecuencia & 2.84 & 0.969 & -0.591 & -0.080 \\
\hline BG7. Se ha sentido nervioso & 2.55 & 0.940 & -0.271 & -0.077 \\
\hline BG8. Su salud es peor comparada con la de hace un año & 2.50 & 1.023 & -0.363 & -0.510 \\
\hline BG9. Siente que se cansa con facilidad & 2.30 & 1.001 & -0.227 & -0.257 \\
\hline BG10. Se siente triste sin un motivo aparente & 2.51 & 1.197 & -0.326 & -0.840 \\
\hline BG11. Siente que no puede dormir & 2.68 & 1.027 & -0.441 & -0.275 \\
\hline BG12. Siente que no tiene hambre & 2.81 & 1.108 & -0.620 & -0.457 \\
\hline BG13. Se enoja fácilmente & 2.60 & 1.071 & -0.422 & -0.384 \\
\hline BG14. Asistió al médico solo cuando se sintió enfermo & 1.15 & 1.192 & 0.937 & -0.041 \\
\hline \multicolumn{5}{|l|}{ Dificultad percibida para: } \\
\hline AUT1. Bañarse & 3.40 & 1.054 & -1.830 & $2.448^{*}$ \\
\hline AUT2. Salir a la calle para pasear & 3.12 & 1.225 & -1.242 & 0.356 \\
\hline AUT3. Vestirse & 3.40 & 1.022 & -1.812 & $2.543^{*}$ \\
\hline AUT4. Salir a hacer pagos de servicios & 3.03 & 1.277 & -1.134 & 0.076 \\
\hline AUT5. Limpiar su casa o cuarto & 3.08 & 1.230 & -1.186 & 0.266 \\
\hline AUT6. Salir de compras & 2.96 & 1.321 & -1.027 & -0.243 \\
\hline AUT7. Cocinar & 3.07 & 1.235 & -1.165 & 0.178 \\
\hline
\end{tabular}

*No se consideraron para el AFE y AFC. $M=$ media aritmética, $D E=$ desviación estándar, $A s=a s i m e t r i ́ a, ~ K=c u r t o s i s$.

Fuente: elaboración propia. 
adecuados. Además, el índice de replicabilidad del constructo para cada factor extraído fue de $\mathrm{H}_{\mathrm{F} 1}=0.975, \mathrm{H}_{\mathrm{F} 2}=0.857 \mathrm{H}_{\mathrm{F} 3}=0.866$ y $\mathrm{H}_{\mathrm{F} 4}=0.858$. Por lo que se optó por mantener la solución de cuatro componentes.

En dicha solución, la varianza total explicada para la solución de cuatro factores fue de $62.84 \%$, de las 21 variables incluidas originalmente, se integraron 17 en la estructura, quedaron fuera dos reactivos debido a un alto coeficiente de asimetría y curtosis, tres indicadores más no obtuvieron una carga factorial suficiente. El resto de las variables presentaron una asimetría inferior a dos puntos $y$ un perfil tendiente a la mesocurtosis; sin embargo, en todas las variables se rechazó la hipótesis nula con la prueba de Kolmogorov-Smirnov, que indica que no existe evidencia de distribución normal. En los indicadores de autonomía, la media en la mayoría de las variables se ubicó en un nivel de poca dificultad, según las opciones de respuesta, para el bienestar, los puntajes corresponden a la opción de respuesta a veces.

El primer factor en la estructura corresponde a la dimensión de Autonomía, mismo que explica el $37.20 \%$ de la varianza total, tiene un coeficiente alfa de Cronbach de 0.941 y una correlación promedio inter-elementos de 0.762 . Este factor mide con cinco reactivos el nivel de limitación percibida por el adulto mayor para realizar actividades instrumentales de la vida cotidiana. En la composición original

Tabla 2.

Matriz de cargas factoriales y coeficientes de discriminación para la Autoevaluación Breve de Bienestar y Autonomía en Adultos Mayores

\begin{tabular}{|c|c|c|c|c|c|}
\hline \multirow{2}{*}{ Variables } & \multicolumn{4}{|c|}{ Factor } & \multirow[b]{2}{*}{$C D$} \\
\hline & AUT & $\mathrm{BF}$ & $\mathrm{BE}$ & BS & \\
\hline AUT4. Salir a hacer pagos de servicios & 0.940 & & & & 0.880 \\
\hline AUT6. Salir de compras & 0.935 & & & & 0.885 \\
\hline AUT2. Salir a la calle para pasear & 0.880 & & & & 0.854 \\
\hline AUT5. Limpiar su casa o cuarto & 0.819 & & & & 0.831 \\
\hline AUT7. Cocinar & 0.731 & & & & 0.756 \\
\hline BG3. Su salud física & & 0.713 & & & 0.706 \\
\hline BG6. Se enferma con mayor frecuencia & & 0.657 & & & 0.512 \\
\hline BG1. Su estado de salud en general & & 0.655 & & & 0.669 \\
\hline BG9. Siente que se cansa con facilidad & & 0.620 & & & 0.560 \\
\hline BG8. Su salud es peor comparada hace un año & & 0.573 & & & 0.589 \\
\hline BG12. Siente que no tiene hambre & & & 0.762 & & 0.580 \\
\hline BG10. Se siente triste sin motivo aparente & & & 0.664 & & 0.551 \\
\hline BG7. Se ha sentido nervioso & & & 0.561 & & 0.535 \\
\hline BG11. Siente que no puede dormir & & & 0.533 & & 0.499 \\
\hline BG5. Su relación con familiares & & & & 0.750 & 0.538 \\
\hline BG2. Su estado de ánimo & & & & 0.651 & 0.654 \\
\hline BG4. Sus relaciones sociales & & & & 0.544 & 0.601 \\
\hline
\end{tabular}

Método de extracción: cuadrados mínimos no ponderados. Método de rotación: Promax con normalización Kaiser. a. La rotación ha convergido en 6 iteraciones. $A U T=$ Autonomía, $B F=$ Bienestar físico, $B E=$ Bienestar emocional, $B S=$ Bienestar social, $C D=C o e f i c i e n-$ te de discriminación.

Fuente: elaboración propia. 
del factor era de siete reactivos, mismos que no obtuvieron la carga factorial inferior a 0.40 (Bandalos y Finney, 2010; Izquierdo, Olea, y Abad, 2014).

El segundo factor, se etiquetó como Bienestar físico, explicó el $11.64 \%$ de la varianza, presentó una alfa de Cronbach de 0.811 y correlación inter-elementos de 0.474. Este factor representa la percepción positiva o negativa del estado de salud física, actual y durante el último año, así como la sensación de deterioro físico, contiene cinco reactivos en su estructura.

Por otro lado, el tercer factor es el Bienestar emocional, tuvo un porcentaje de varianza explicada de $7.70 \%$, midió con cuatro reactivos el efecto de las emociones negativas en la percepción de bienestar. El alfa de Cronbach de este factor fue de 0.745 , con una correlación promedio inter-elementos de 0.425 .

Por último, el cuarto factor etiquetado como Bienestar social, explicó el $6.28 \%$ de la varianza, se compone de tres reactivos que midieron la satisfacción emocional del adulto mayor con la forma en que lleva sus relaciones sociales y familiares. Este factor obtuvo un coeficiente alfa de Cronbach de 0.763 , la correlación promedio inter-elementos fue de 0.523 .

Hay que destacar que los factores de bienestar originalmente se integraron en una sola dimensión denominada bienestar general, misma que incluía 14 indicadores. Al sumar los reactivos de las tres dimensiones de bienestar obtenidas, se encontró una reducción de dos reactivos en la estructura del factor propuesto hipotéticamente.

Los índices de discriminación para 17 reactivos que integran la Autoevaluación Breve de Bienestar y Autonomía en Adultos Mayores resultaron con valores superiores a 0.30 y con signo positivo, lo que muestra una adecuada coherencia interna.

El AFC se realizó con una estructura correlacionada, si bien las dimensiones de bienestar se

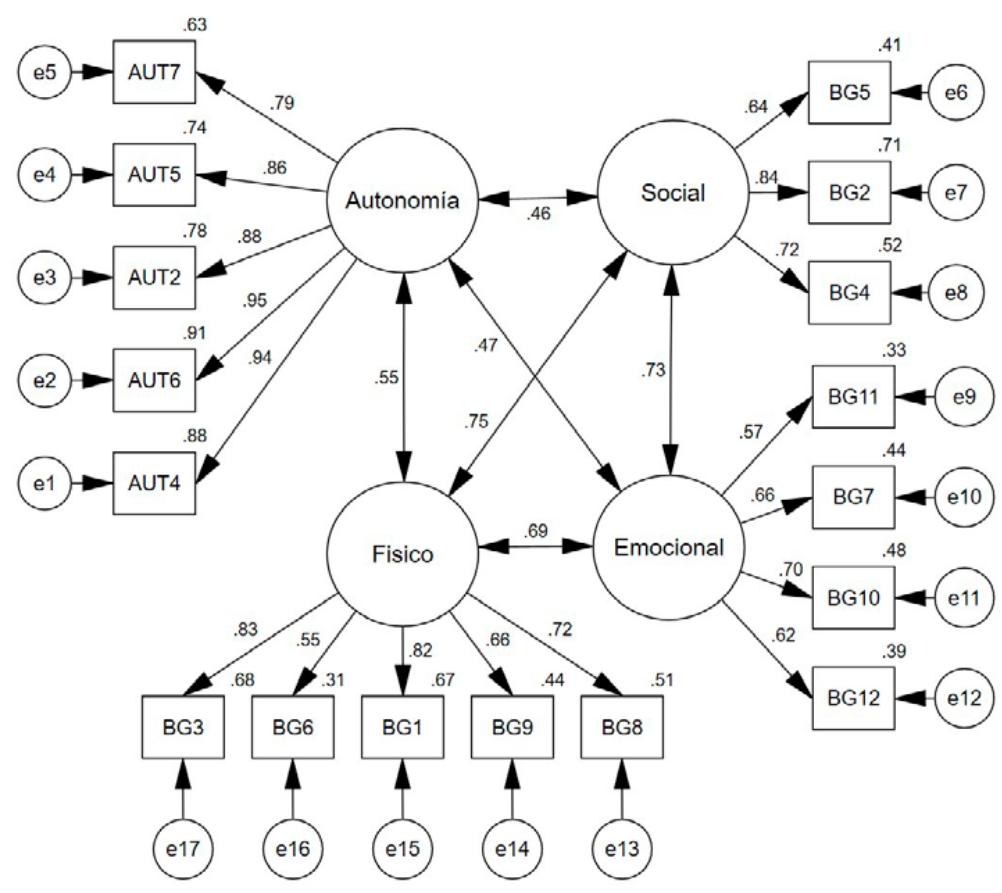

Figura 1. Diagrama del Análisis Factorial Confirmatorio con parámetros estandarizados por el Método de Mínimos Cuadrados Generalizados para la muestra conjunta.

Fuente: elaboración propia. 
Tabla 3.

Índices de bondad de ajuste para tres modelos de AFC divididos por muestra

\begin{tabular}{|c|c|c|c|}
\hline Índice & Conjunta & Yucatán & Coahuila \\
\hline RMR & 0.128 & 0.164 & 0.150 \\
\hline GFI & 0.918 & 0.876 & 0.918 \\
\hline AGFI & 0.890 & 0.835 & 0.891 \\
\hline RMSEA & 0.071 & 0.080 & 0.065 \\
\hline Alfa de Cronbach & 0.884 & 0.877 & 0.906 \\
\hline Alfa Ordinal $(\omega)$ & 0.943 & 0.951 & 0.984 \\
\hline Correlación promedio inter-elementos & 0.286 & 0.272 & 0.337 \\
\hline
\end{tabular}

Nota: GFI= Índices de Bondad de Ajuste General de Jöreskog. AGFI= Índice de Bondad de Ajuste Corregido de Jöreskog. $R M S E A=$ Error Cuadrático Medio de Aproximación. RMR= Raíz del Residuo Estandarizado.

Fuente: elaboración propia.

correlacionaron de una manera importante entre sí, destaca el relativo equilibrio en las correlaciones con la dimensión de autonomia del adulto mayor, principalmente del bienestar físico. El modelo factorial confirmado preservó la misma cantidad de parámetros que el modelo hipotetizado mediante el AFE. Para la muestra conjunta el ajuste del RMR fue relativamente lejano a cero, que es el valor de un ajuste muy bueno. Sin embargo, los índices de ajuste de Jöreskog reportaron valores de 0.90 , además el valor de RMSEA fue inferior a 0.075, mismo que se considera aceptable. Esta estructura enconjunto obtuvo un coeficiente alfa de Cronbach aceptable

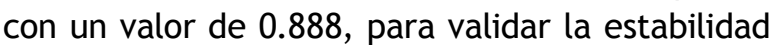
de este valor, se obtuvo elvalor del coeficiente alfa ordinal, que reportó un puntaje de 0.943 , la correlación promedio entre reactivos fue de 0.286 , que demuestra coherencia interna en la estructura.

Por otro lado, el mismo modelo hipotetizado para la muestra conjunta se probó para la muestra de Yucatán y la de Coahuila. La muestra de Yucatán presentó un ajuste ligeramente baja en comparación con la de Coahuila, ya que los índices de Jöreskog no superaron el criterio de 0.90 tanto el índice general como el corregido. Los coeficientes de alfa de Cronbach y alfa Ordinal fueron aceptables en ambas muestras.

\section{Discusión}

El aumento en la esperanza de vida y la cantidad de adultos mayores en el mundo requiere de un seguimiento adecuado de las condiciones en que este grupo de población vive y se desarrolla, lo que permita dar un contrasentido a la perspectiva incierta de sus condiciones cualitativas así como la adecuada atención a las nuevas exigencias y necesidades que los sistemas sociales habrán de atender, como lo han afirmado diversos autores (Lolas Stepke, 2001; Manrique-Espinoza et al., 2013; Vivaldi y Barra, 2012).

Se ha mencionado que, la dimensión afectiva o emocional, ha sido subestimada en los modelos explicativos del bienestar del adulto mayor, centrados principalmente en las consideraciones de desempeño físico, en este estudio, uno de los factores que se configuró dentro de la escala obedece a la necesidad de valorar el impacto de las emociones sobre el funcionamiento cotidiano, explorado mediante indicadores asociados a la depresión, mismos que se han correlacionado con una mayor morbilidad en el adulto mayor (Afilalo et al., 2012; Chen y Chen, 2017).

Además, se ha mostrado que impacta de manera negativa la situación funcional y social del adulto mayor (Domínguez-Ardila y García-Manrique, 2014), 
hipótesis que se confirma con los resultados de este trabajo al identificar las correlaciones entre el factor que mide el bienestar emocional y la percepción de autonomía.

Adicionalmente, el concepto de bienestar forma parte de la naturaleza y definición de la calidad de vida general y relacionada con la salud, es complementario a las mediciones clínicas del desempeño físico y mental del adulto mayor, lo que aporta a un mayor conocimiento de la percepción sobre su estado de salud (Bernal Reyes et al., 2015; Cabezas-León et al., 2008). Este instrumento cumple el propósito de aportar variables a la clinimetría del adulto mayor, principalmente en las variables psicológicas y sociales, lo que responde al interés internacional sobre la medición y evaluación de la calidad de vida y el bienestar en este grupo de la población (Haywood et al., 2005; Zaninotto et al., 2009).

Por otro lado, las limitaciones del estudió radican en la falta de una medición paralela para la validez de criterio de la escala propuesta, ya que en inicio no era uno de los objetivos del estudio, sin embargo es importante que, en aplicaciones subsecuentes se incluyan variables criterio para dar mayor validez y capacidad de replicabilidad a los constructos obtenido, aunque los indicadores de calidad de los factores calculados con el AP resultaron óptimos y con poca probabilidad de cambio en mediciones posteriores en otras muestras.

\section{Conclusiones}

Sin que denote prejuicio, la fragilidad y vulnerabilidad correlacionada con el ascenso de edad implica aparente disminución en las actividades instrumentales y sociales de la vida cotidiana por eso en este estudio se decidió utilizar una autoevaluación de la limitación física para dichas actividades, además que, la percepción de bienestar se ve influida por elementos culturales (Melguizo Herrera et al, 2012). Además que, cada adulto mayor vive cambios individuales en sus capacidades físicas, sociales y mentales que es necesario explorar y relacionar con demás aspectos de su vida (Loredo-Figueroa et al., 2016).

La estructura que se identificó en ambas muestras presentó indicadores de ajuste estadístico adecuado. Originalmente, la estructura consideraba dos dimensiones solamente: la autonomía y el bienestar, sin embargo, los resultados mostraron la separación en tres dimensiones de la medición de bienestar. Estos datos dan evidencia de validez de constructo de la medición de autonomía y bienestar en los adultos mayores entrevistados.

Por otro lado, la estructura factorial definida con la escala propuesta es congruente con las variables consideradas en las definiciones que existen sobre el bienestar en el envejecimiento exitoso a nivel internacional, que incluyen aspectos como la satisfacción vital, la satisfacción con la vida cotidiana y la presencia de emociones positivas (Carmona Valdés y Ribeiro Ferreira, 2010), la estructura propuesta considera de manera armónica las dimensiones físicas, sociales y emocionales en el adulto mayor, elementos relacionados también con una existencia agradable y una vida en condiciones cualitativamente satisfactorias (Pérez Vargas, 2015).

\section{Referencias}

Acevedo Alemán, J. y González Tovar, J. (2014). No envejecemos igual: la religiosidad y el género en adultos mayores del noreste de méxico. Reflexiones, 93(1), 133-144.

Afilalo, J., Mottillo, S., Eisenberg, M. J., Alexander, K. P., Noiseux, N., Perrault, L. P., . . Bergman, H. (2012). Addition of Frailty and Disability to Cardiac Surgery Risk Scores Identifies Elderly Patients at High Risk of Mortality or Major Morbidity. Circulation: Cardiovascular Quality and Outcomes, 5(2), 222.

Alvarado-García, A., Lamprea-Reyes, L. y MurciaTabares, K. (2017). La nutrición en el adulto mayor: una oportunidad para el cuidado de enfermería. Enfermería universitaria, 14(3), 199-206. https://dx.doi.org/10.1016/j. reu.2017.05.003.

Bandalos, D. L. y Finney, S. J. (2010). Factor analysis: Exploratory and confirmatory. In G. R. Hancock y R. O. Mueller (Eds.), The reviewer's guide to quantitative methods in the Social Sciences (pp. 93-114). New York: Routledge. 
Bentler, P. M. (1977). Factor simplicity index and transformations. Psychometrika, 42(2), 277295. https://doi.org/10.1007/bf02294054

Bernal Reyes, N., Arias Díaz, A., Hormigó Puertas, I. y Roselló Leyva, A. (2015). Actividades de la vida diaria y calidad de vida en adultos mayores operados de catarata. Revista Mexicana de Oftalmología, 89(3), 141-149. https://doi. org/10.1016/j.mexoft.2014.09.005

Borda, M. G., Acevedo González, J. C., David, D. G., Morros González, E. y Cano, C. A. (2016). Dolor en el anciano: calidad de vida, funcionalidad y factores asociados. Estudio SABE, Bogotá, Colombia. Revista Española de Geriatría y Gerontología, 51(3), 140-145. http://dx.doi. org/10.1016/j.regg.2015.07.001

Botero de Mejía, B. E. y Pico Merchán, M. E. (2007). Calidad de vida relacionada con la salud (CVRS) en adultos mayores de 60 años: una aproximación teórica. Hacia la Promoción de la Salud, 12, 11-24.

Cabezas-León, M., García-Caballero, J. y MorenteMatas, P. (2008). Impacto de la cirugía de catarata: agudeza visual y calidad de vida. Archivos de la Sociedad Española de Oftalmología, 83, 237-248.

Cardona, A. D. (2010). Comparativo de la calidad de vida del adulto mayor. Medellín, 2008. Revista Facultad Nacional de Salud Pública, 28, 149-160.

Carmona Valdés, S. E. y Ribeiro Ferreira, M. (2010). Actividades sociales y bienestar personal en el envejecimiento. Papeles de población, 16, 163-185.

Cervantes Becerra, R. G., Villarreal Ríos, E., Galicia Rodríguez, L., Vargas Daza, E. R., y Martínez González, L. (2015). Estado de salud en el adulto mayor en atención primaria a partir de una valoración geriátrica integral. Atención Primaria, 47(6), 329-335. https://doi. org/10.1016/j.aprim.2014.07.007

Chang, H.-T., Liu, L.-F., Chen, C.-K., Hwang, S.-J., Chen, L.-K. y Lu, F.-H. (2010). Correlates of institutionalized senior veterans' quality of life in Taiwan. Health and Quality of Life Outcomes, 8, 70-70. doi:10.1186/1477-7525-8-70

Chen, H.-M. y Chen, C.-M. (2017). Factors Associated with Quality of Life Among Older Adults with Chronic Disease in Taiwan. International Journal of Gerontology, 11(1), 12-15. https:// doi.org/10.1016/j.ijge.2016.07.002

Damián, A. (2016). Social security, pensions and old age adults' poverty in Mexico. Acta Sociológica, 70, 151-172. https://doi.org/10.1016/j. acso.2017.01.007

Domínguez-Ardila, A. y García-Manrique, J. G. (2014). Valoración geriátrica integral. Atención Familiar, 21(1), 20-23. https://doi.org/10.1016/ S1405-8871(16)30006-2

Fleming, J. S. y Merino Soto, C. (2005). Medidas de simplicidad y de ajuste factorial: un enfoque para la evaluación de escalas construidas factorialmente. Revista de Psicologia de la PUCP, 23(2), 251-266.

García, C. E. (2010). Algunas estrategias de prevención para mantener la autonomía y funcionalidad del adulto mayor. Revista Médica Clínica Las Condes, 21(5), 831-837. https://doi. org/10.1016/S0716-8640(10)70604-0

Garza Sánchez, R. I. y González Tovar, J. (2017). Comparativo de la calidad de vida subjetiva entre México y Chile: perspectiva desde el envejecimiento. 31(123), 15. https://doi. org/10.15517/ap.v31i123.28543

González Tovar, J. y Garza Sánchez, R. I. (2016). Primera revisión del Cuestionario de Calidad de Vida para Adultos Mayores en Comunidad CCVAMC-62. Ciencia Ergo Sum.

Guerrero-López, J. B., Mendieta Cabrera, D., LaraMuñoz, M. d. C. y Ortiz García, R. (2017). Evaluación de la calidad de vida y depresión en pacientes con artritis reumatoide en un hospital general. Revista Colombiana de Reumatología, 24(4), 199-204. https://doi. org/10.1016/j.rcreu.2017.05.008 
Haywood, K. L., Garratt, A. M. y Fitzpatrick, R. (2005). Quality of life in older people: a structured review of generic self-assessed health instruments. Quality of Life Research, 14(7), 1651-1668. https://doi.org/10.1007/ s11136-005-1743-0

Hernández Sampíeri, R., Fernández Collado, C. y Baptista Lucio, P. (2014). Metodología de la Investigación (Sexta edición ed.). Ciudad de México: McGrawHill.

Izquierdo, I., Olea, J. y Abad, F. J. (2014). Exploratory factor analysis in validation studies: Uses and recommendations. Psicothema, 26(3), 395-400.

Jiménez-Aguilera, B., Baillet-Esquivel, L. E., Ávalos-Pérez, F. y Campos-Aragón, L. (2016). Dependencia funcional y percepción de apoyo familiar en el adulto mayor. Atención Familiar, 23(4), 129-133. http://dx.doi.org/10.1016/j. af.2016.08.002

Karlsson, M. K., Magnusson, H., von Schewelov, T. y Rosengren, B. E. (2013). Prevention of falls in the elderly-a review. Osteoporosis International, 24(3), 747-762. http://dx.doi. org/10.1007/s00198-012-2256-7

Lloret-Segura, S., Ferreres-Traver, A., HernándezBaeza, A. y Tomás-Marco, I. (2014). El Análisis Factorial Exploratorio de los Ítems: una guía práctica, revisada y actualizada. Anales de Psicología, 30, 1151-1169. http://dx.doi. org/10.6018/analesps.30.3.199361

Lolas Stepke, F. (2001). Las dimensiones bioéticas de la vejez. Acta bioethica, 7, 57-70. http:// dx.doi.org/10.4067/S1726-569X2001000100005

Loredo-Figueroa, M. T., Gallegos-Torres, R. M., Xeque-Morales, A. S., Palomé-Vega, G. y Juárez-Lira, A. (2016). Nivel de dependencia, autocuidado y calidad de vida del adulto mayor. Enfermería Universitaria, 13(3), 159-165. https://doi.org/10.1016/j.reu.2016.05.002

Lorenzo-Seva, U. (2003). A factor simplicity index. Psychometrika, 68(1), 49-60. https://doi. org/10.1007/bf02296652
Manrique-Espinoza, B., Salinas-Rodríguez, A., Moreno-Tamayo, K. M., Acosta-Castillo, I., Sosa-Ortiz, A. L., Gutiérrez-Robledo, L. M. y Téllez-Rojo, M. M. (2013). Condiciones de salud y estado funcional de los adultos mayores en México. Salud Pública de México, 55, S323-S331.

Marín Monroy, C. y Castro Molinares, S. (2011). Adulto mayor en Santa Marta y calidad de vida. Memorias, 9(16), 120-129. http://dx.doi. org/10.16925/issn.0124-4361

Maseda, A., Diego-Diez, C., Lorenzo-López, L., López-López, R., Regueiro-Folgueira, L. y Millán-Calenti, J. C. (2018). Quality of life, functional impairment and social factors as determinants of nutritional status in older adults: The VERISAÚDE study. Clinical Nutrition, 37(3), 993-999. https://doi.org/10.1016/j. clnu.2017.04.009

Mazzanti Di Ruggiero, M. d. I. Á. (2011). Declaración de Helsinki, principios y valores bioéticos en juego en la investigación médica con seres humanos. Revista Colombiana de Bioética, 6(1), 125-144.

Melguizo Herrera, E., Acosta López, A. y Castellano Pérez, B. (2012). Factores asociados a la calidad de vida de adultos mayores. Cartagena (Colombia). Revista Salud Uninorte, 28(2), 261-268.

Monroy-Rojas, A., Contreras-Garfias, M. E., GarcíaJiménez, M. A., García-Hernández, M. L., Cárdenas-Becerril, L. y Rivero-Rodríguez, L. F. (2016). Estatus funcional de adultos mayores de Tláhuac, Ciudad de México. Enfermería Universitaria, 13(1), 25-30. https://doi. org/10.1016/j.reu.2016.01.005

Oliden, P. E. y Zumbo, B. D. (2008). Reliability coefficients for ordinal response scales. Psicothema, 20(4), 896-901.

Pelcastre-Villafuerte, B. E., Treviño-Siller, S., González-Vázquez, T. y Márquez-Serrano, $M$. (2011). Apoyo social y condiciones de vida de adultos mayores que viven en la pobreza urbana en México. Cadernos de Saúde Pública, 
27(3), 460-470. http://dx.doi.org/10.1590/ S0102-311X2011000300007

Pérez Vargas, I. (2015). Calidad de vida en ancianos institucionalizados en México: retos. Tiempo, 18(32), $1-2$.

Ramírez-Villada, J. F., Chaparro-Obando, D., LeónAriza, H. H. y Salazar Pachón, J. (2015). Efecto del ejercicio físico para el control de los factores de riesgo cardiovascular modificables del adulto mayor: revisión sistemática. Rehabilitación, 49(4), 240-251. https://doi. org/10.1016/j.rh.2015.07.004

Rodriguez, A., Reise, S. P. y Haviland, M. G. (2016). Applying Bifactor Statistical Indices in the Evaluation of Psychological Measures. Journal of Personality Assessment, 98(3), 223-237. https://doi.org/10.1080/00223891.2015.1089249

Salazar Pachón, J. D., Ramírez Villada, J. F., Chaparro, D. y León, H. H. (2014). Revisión sistemática sobre el impacto de la actividad física en los trastornos de la marcha en el adulto mayor. Apuntsuntes en Educación Física y Deportes,4 (118), 30-39. https://doi.org/10.5672/apunts.2014-0983. es.(2014/4).118.03

Sève-Ferrieu, N. (2017). Independencia, autonomía y calidad de vida: análisis y evaluaciones. EMC-Kinesiterapia-Medicina Física, 38(1), 1-16. https://doi.org/10.1016/S1293-2965(16)81752-1

Timmerman, M. E. y Lorenzo-Seva, U. (2011). Dimensionality assessment of ordered polytomous items with parallel analysis. Psychology Methods, 16(2), 209-220. https:// doi.org/10.1037/a0023353

Urzúa, A. y Caqueo-Urízar, A. (2012). Calidad de vida: Una revisión teórica del concepto.
Terapia psicológica, 30(1), 61-71. 10.4067/ S0718-48082012000100006

Urzúa, A., Pavlov, R., Cortés, R. y Pino, V. (2011). Factores Psicosociales Relacionados con la Calidad de Vida en Salud en Pacientes Hemodializados. Terapia psicológica, 29(1), 135-140.

Urzúa, M. A. y Navarrete, M. (2013). Calidad de vida en adultos mayores: análisis factoriales de las versiones abreviadas del WHOQoL-Old en población chilena. Revista médica de Chile, 141(1), 28-33.

Vitorino, L., Lucchetti, G., Santos, A., L G Lucchetti, A., Ferreira, E., Adami, N. y Vianna, L. (2016). Spiritual Religious Coping is Associated with Quality of Life in Institutionalized Older Adults. Journal of religion and health, 55(2), 549-559. https://doi.org/10.1007/s10943-015-0148-9

Vivaldi, F. y Barra, E. (2012). Bienestar psicológico, apoyo social percibido y percepción de salud en adultos mayores. Terapia psicológica, 30(2), 23-29. http://dx.doi.org/10.4067/ S0718-48082012000200002

Zaninotto, P., Falaschetti, E. y Sacker, A. (2009). Age trajectories of quality of life among older adults: Results from the English Longitudinal Study of Ageing, 18(10), 1301-1309. https://doi. org/10.1007/s11136-009-9543-6

Zheng, G., Huang, M., Liu, F., Li, S., Tao, J. y Chen, L. (2015). Tai Chi Chuan for the Primary Prevention of Stroke in Middle-Aged and Elderly Adults: A Systematic Review. Evidence-Based Complementary and Alternative Medicine, 2015, 1-18. http://dx.doi.org/10.1155/2015/742152 